\title{
Spectral Characteristics of the Annual Mean Rainfall Series in Ghana
}

\author{
K. Oduro-Afriyie ${ }^{1 *}$ and D. C. Adukpo ${ }^{2}$ \\ ${ }^{1}$ Department of Physics, University of Ghana, Legon-Accra, Ghana \\ ${ }^{2}$ Department of Physics, University of Cape Coast, Cape Coast, Ghana \\ *Corresponding author, E-mail: koduro@ug.edu.gh
}

\begin{abstract}
Time series of standardised (normalised) deviations of annual mean rainfall in Ghana for the period 1961-1998 was plotted using data for 30 stations. The plot revealed widespread floods in 1962, 1963 and 1996, and widespread droughts in 1977, 1983, 1992 and 1998. To check for periodicities, the time series was subjected to a power spectrum analysis using the Maximum Entropy Spectral Analysis (MESA) technique. A number of periodicities were detected. A periodicity of 5.6 years was found to be highly significant. That of 2.7 years was of borderline significance. The rest of the periodicities were insignificant. The most recent floods or excess rains (positive deviation) within the 1961-1998 period occurred in 1996. The next possible floods were, therefore, predicted to occur during the year 2001 or 2002. The widespread floods in Ghana during the period have proved this prediction right. The next major floods in Ghana are likely to occur in year 2006 or 2007.
\end{abstract}

\section{Introduction}

Rainfall patterns usually have spatial and temporal variabilities. These variabilities affect the agricultural production, water supply, transportation, the entire economy of a region, and the existence of its people. In regions where the year-to-year variability is high, people often suffer great calamities due to floods or droughts. Whereas damage due to extremes of rainfall cannot be avoided completely, a forewarning could certainly be useful (Nicholls, 1980).

Methods of prediction of rainfall extremes have often been based on studies of physical effects of rainfall or on statistical studies of rainfall time series. Lansford (1977) reported a study of tree rings in USA and concluded that a 20-22 year periodicity was evident, indicating a possible drought in the 1990s. From historical records of rainfall and general atmospheric circulation patterns in different parts of the world, Winstanley (1973a, 1973b) noticed a 200-year cycle and concluded that a severe drought could occur in about 2030 AD in Sahel and northwestern India.

In recent years, annual rainfall series have been studied for detection of periodicities mostly by the autocorrelation method of Blackman \& Tukey (1958). Nicholson \& Entekhabi (1986) conducted a detailed power spectrum analysis of African rainfall series using Blackman-Tukey and Fourier methods. Their analysis revealed quasi-periodicities clustered in four bands at 2.2-2.4, 2.6-2.8, 3.3-3.8 and 5.0-6.3 years, common throughout equatorial and southern Africa but only weakly evident in northern Africa. Other workers (Tabony, 1979; Kane \& Trivedi, 1982) have used the sophisticated method of Maximum Entropy Spectral Analysis (MESA), formulated by Burg (1967) and reviewed by Ulrych \& Bishop (1975). Periodicities ranging from 2-3 years (Quasi-Biennial Oscillation) to several tens of years with varying amplitudes have been reported for various regions. Many workers are inclined to conclude that the series are random or quasi-random and quasistationary (Winstanley, 1973a, Nicholson, 1980).

The purpose of this study is to determine the characteristics of oscillations that appear in the time series of annual rainfall amounts in Ghana with a view to predicting rainfall extremes in the country. The analysis is carried out using the Maximum Entropy Spectral Analysis (MESA) technique. The results of the analysis could provide informa-tion that would be helpful in formulating policies to mitigate the problems of soil erosion, flooding, drought and bushfires in Ghana.

Periodicities and prediction

\section{Materials and methods}




\section{West Africa Journal of Applied Ecology (WAJAE) -ISSN: 0855-4307 \\ Volume 9 (Jan - Jun 2006) \\ www.wajae.org}

Trends and periodicities in African rainfall have been studied by Bunting et al. (1976) for West Africa; Rodhe \& Virji (1976) for East Africa; Tyson et al. (1975) for South Africa and Ogallo (1979) and Nicholson \& Entekhabi (1986) for various parts of Africa. Whereas no significant trends were detected in Africa up to about 1965, significant quasi-periodicities have been reported clustered in the bands 2.2-2.4, 2.6-2.8 3.3-3.8, 5.0-6.3 years (Nicholson \& Entekhabi, 1986). Kane \& Trivedi (1986) report significant periodicities for some African regions, notably Sahel and South Africa, for data up to the mid 70s. Since then, some regions (e.g. West Africa) have suffered drastic changes like prolonged droughts. They, therefore, conducted a power spectrum analysis for the series reported by Nicholson (1994) and one series of Hulme (1994) for south-western Africa. They used MESA, which detects spectral peaks very accurately. Since amplitude estimations are not reliable in MESA (Kane \& Trivedi, 1982), they obtained the peaks $T_{k}(k=1$ to $n)$ from MESA and used the same in the expression:

where $f(t)$ is the observed time series and $E$ is the error factor.

The parameters $A_{0}, A_{k}, B_{k}$ and their standard errors were estimated by conduc-ting a multiple regression analysis (MRA) (Bevington, 1969). From these, one can obtain the amplitudes, $\mathrm{R}_{\mathrm{k}}$, and their standard error $\sigma$ which is common to all $\mathrm{R}$ in this methodology. Amplitudes greater than $2 \sigma$ are significant at 95\% (a priori) con-fidence level. Their corresponding perio-dicities are, therefore, also significant. Amplitudes equal to $2 \sigma$ are said to be of borderline signifi-cance. Their corresponding periodicities are likewise of borderline significance. Amplitudes less than $2 \sigma$ are insignificant at $95 \%$ confidence level. Their corresponding perio-dicities are likewise insignificant.

MRA probably inflates significantly by over-fitting. Again, there is no guarantee that these peaks in the future will have the same relative proportions. Even for Southern Oscillation Index (SOI), Wright (1977) demonstrated that the spectra varied considerably for four intervals 1851-1882, 1881-1912, 1911-1942 and 1941-1972. Similar changes could have occurred for the rainfall series and may occur in future. As such, prediction possibilities seem to be bleak. Using a 7-year running mean of annual Senegal river runoff, Faure \& Gac (1981) demonstrated a 30-year cycle in sahelian rainfall and predicted a return to wetter conditions by 1985. This did not come true (Kane \& Trivedi, 1986). Their prediction for Sahel also failed. For the northeastern part of South Africa, Kane \& Trivedi (1986) had predicted below average rainfall for 1982-87, which seems to have come true. It, therefore, seems that among all these series, South Africa has the best chance of having some prediction potential.

\section{Spectral analysis}

Spectral analysis is a method used in estimating spectral density function or spectrum of a given time series. It is essentially a modification of Fourier analysis so as to make it suitable for stochastic rather than deterministic functions of time. Harmonically, the cyclic components of a time series can be determined using the Fourier series under very general conditions for any function $U_{t}$. Thus for a time series $\left(U_{t}\right)$ with period of oscillation T, we have 


$$
\begin{aligned}
& U_{t}=A_{o}+A_{1} \sin \frac{2 \pi t}{T}+A_{2} \sin \frac{2 \pi \cdot 2 t}{T}+\ldots+B_{1} \cos \frac{2 \pi t}{T}+B_{2} \cos \frac{2 \pi \cdot 2 t}{T}+\ldots \\
& U_{t}=A_{o}+A_{1} \sin \frac{2 \pi t}{T}+A_{2} \sin \frac{2 \pi \cdot 2 t}{T}+\ldots+B_{1} \cos \frac{2 \pi t}{T}+B_{2} \cos \frac{2 \pi \cdot 2 t}{T}+\ldots
\end{aligned}
$$

where

$$
\begin{array}{ll}
A_{i}=\frac{2}{n} \sum_{t=1}^{n} U_{t} \cdot \sin \left(\frac{2 \pi}{T} . i t\right), & (\mathrm{i}=1,2, \ldots) \\
B_{i}=\frac{2}{n} \sum_{t=1}^{n} U_{t} \cdot \cos \left(\frac{2 \pi}{T} . i t\right), & (\mathrm{i}=1,2, \ldots) \\
A_{o}=\frac{1}{n} \sum_{t=1}^{n} U_{t} &
\end{array}
$$

and $\mathrm{n}$ is the number of terms in the time series. For instance, if the period of oscillation is 12 months and $\mathrm{U}_{1}, \mathrm{U}_{2}$ $\ldots U_{12}$ is the series or average of series for a number of years, the constants, $A_{i}$ and $B_{i}$, are given by

$$
\begin{gathered}
A_{o}=\frac{1}{12} \sum_{t=1}^{12} U_{t} \\
A_{i}=\frac{2}{12} \sum_{t=1}^{12} U_{t} \cdot \sin \left(\frac{2 \pi}{12} \cdot i t\right) \\
B_{i}=\frac{2}{12} \sum_{t=1}^{12} U_{t} \cdot \cos \left(\frac{2 \pi}{12} \cdot i t\right)
\end{gathered}
$$$$
(i=1,2 \ldots 5)
$$

A major drawback of harmonic analysis lies in huge calculations. It is in view of this drawback that the Maximum Entropy Spectral Analysis is adopted in this study to pick the periodicities inherent in the time series data (rainfall figures).

Maximum entropy spectral analysis (MESA)

Maximum Entropy Method (MEM) detects periodicities much more accurately than the conventional BT (Blackman \& Tukey, 1958) method (Burg, 1967; Ulych \& Bishop, 1975). Similar to the parameter lag m in BT, MEM has a parameter called LPEF (Length of the Prediction Error Filter), which can be chosen. With low LPEF, only low periodicities are resolved. Larger LPEF resolve larger periodicities, even those approaching the data length, but the errors are larger and low periodicities show peak-splitting. LPEF $=50 \%$ of the data length generally gives good results (Kane \& Trivedi, 1982).

In BT, only certain frequencies can be used, depending on the lag $\mathrm{m}$, but in MEM, any frequency can be used. The frequency is put in a DO loop and values chosen in regular steps of any size. The frequency range is from 0 


\section{West Africa Journal of Applied Ecology (WAJAE) -ISSN: 0855-4307 \\ Volume 9 (Jan - Jun 2006) \\ www.wajae.org}

to 0.5 , so that periodicities studied are $\mathrm{T}=$ infinity to 2.0 . Note that the minimum period that can be studied is $\mathrm{T}$ $=2.0$ ( 2 year periodicity with annual values series). So the maximum value of frequency $\mathrm{f}$ is 0.5 (this is called folding frequency). One does not go down to $f=0$, but to say $f=0.05(T=20)$. However, in the case where $T$ is used, one puts $\mathrm{T}=2,3,4 \ldots 20$ in a DO loop and a small program finds the corresponding frequencies $\mathrm{f}=1 / 2$, $1 / 3,1 / 4 \ldots 1 / 20$. Power is calculated for these frequencies only. However, in these broad steps of $T$, a periodicity of $\mathrm{T}=2.5$ will be missed. So one can choose finer steps $\mathrm{T}=2.0,2.1,2.2 \ldots 3.0,3.1,3.2 \ldots 20.1,20.2,20.3 \ldots$ Accuracy in higher periodicities (i.e. 20.1, 20.2, etc.) is not required. Hence, one adopts a procedure in which lower periodicities have shorter steps. This is done by putting $\log \mathrm{T}$ instead of $\mathrm{T}$ in the DO loop. The sequence looks as follows:

$\begin{array}{lll}\log T & T & f=(1 / T) \\ & & \\ 0.3010 & 2.00 & 0.500 \text { (folding frequency) } \\ 0.3035 & 2.01 & 0.497 \\ 0.3060 & 2.02 & 0.495 \\ 0.3085 & 2.03 & 0.493 \\ 0.3110 & 2.05 & 0.488 \\ \ldots \ldots & & \\ 0.8010 & 6.32 & 0.158 \\ 0.8035 & 6.36 & 0.157 \\ \ldots \ldots & & \\ 1.3010 & 20.0 & 0.050\end{array}$

The sequence could be terminated at any value of $\mathrm{T}$, say at $\mathrm{T}=30$ or 40 .

The power estimates of MEM are not reliable (Quin et. al., 1987). Hence, MEM is used only to identify the possible periodicities $T_{k}$ which are then used in equation (1). It should be noted, in this case, that MEM and MRA are two independent procedures that are in no way related to each other.

MEM peak detection is very accurate. For example, suppose the data are seasonal values (four values per year) for $\sim 13$ years, i.e., 52 data points. In conventional BT method, with a generally recommended lag $\mathrm{m}$ of $25 \%$ of data length ( $\mathrm{m}=13$ in this case), only certain frequencies $\mathrm{k} / 2 \mathrm{~m}$ can be investigated, i.e. 1/26, 2/26 ... 13/26 (the folding frequency $=0.5$ ). In terms of periodicities, these are $26,13 \ldots 2.36,2.16,2.00$ years. Thus, if the periodicity was 2.25 years, it would be missed. In MEM, there is no such restriction. Any frequency (of course, less than the folding frequency of 0.5) can be investigated and the steps are a matter of choice. Thus, if about 70 steps are investigated between 2.0 and 3.0 years, the periodicities could have a step resolution of 0.01 and a detection accuracy of 0.05 years or better.

MEM resolves peaks even slightly better than the criterion mentioned by Ulrych \& Bishop (1975). Two frequencies, $\mathrm{f}_{1}$ and $\mathrm{f}_{2}$, can be resolved if the data length exceeds

$1 /\left(f_{1}-f_{2}\right)$. Since amplitudes are finally estimated by MRA (MEM is used for locating periodicities $T$ ), the main restriction comes from MRA. Here, the standard error is the same for all periodicities. But, if two perio-dicities are too close, the method gives larger standard errors for both periodicities. In general, a separation of 0.10 (e.g. 2.50 and 2.60) is enough for resolution.

An apprehension usually expressed about MEM is the appearance of spurious peaks. Investigations with artificial (procedure) samples, however, showed no prominent spurious peaks (Kane, 1979). Splitting of periodicity peaks at very large LPEF (approaching the data length) were, however, observed. An LPEF $=50 \%$ of the data length is generally a good and safe compromise (Kane, 1979). 
Data and analysis

Monthly rainfalls for the period 1961-1998 for 30 stations in Ghana were obtained from the Ghana Meteorological Services Department, Accra, Ghana. Fig. 1 shows the map of Ghana with the distribution of these stations. For each station, the annual rainfall values were computed. These values were then used to compute the annual mean rainfall for the period 1961-1998. The deviations of the annual rainfall from the annual mean rainfall for the station were also computed. These deviations were then divided by the standard deviation of the series to obtain the normalised rainfall value for the station. The stations were grouped into the southern zone comprising stations below latitude $8^{\circ} \mathrm{N}$ and the northern zone comprising stations above latitude $8^{\circ} \mathrm{N}$. The grouping was based on the rainy seasonal patterns in Ghana. Stations 1-20 have bimodal rainy seasons while stations 21-30 have monomodal rainy seasons. The data for each zone and also for the country were obtained by averaging the data of the stations they are comprised of. The time series of the normalised rainfall data for the two zones and also for the country were plotted in order to identify possible regular periodicities. 
West Africa Journal of Applied Ecology (WAJAE) -ISSN: 0855-4307

Volume 9 (Jan - Jun 2006)

www.wajae.org

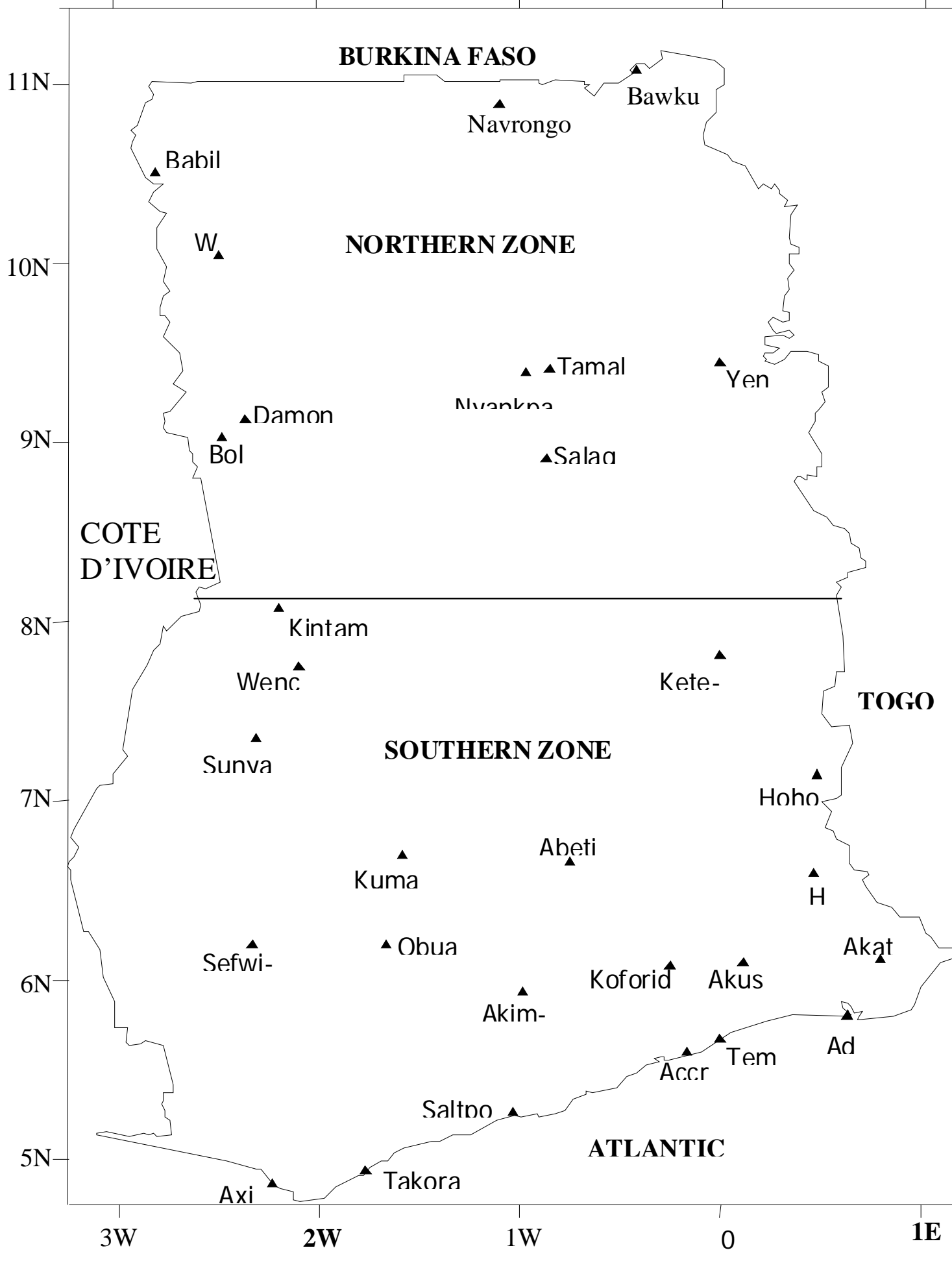

Fig. 1. Map of Ghana showing the distribution of stations considered in this study.

To check for periodicities, however, the time series for the two zones and also for the country were subjected to a power spectrum analysis using the Maximum Entropy Spectral Analysis (MESA) technique. All the probable 


\section{West Africa Journal of Applied Ecology (WAJAE) -ISSN: 0855-4307 \\ Volume 9 (Jan - Jun 2006) \\ www.wajae.org}

LPEFs (i.e. 30\%, 40\%, 50\% and 60\%) worth considering for a data length of 38 years were used in the analysis. However, in line with Kane (1979), only the values obtained for LPEF $=50 \%$ were used.

Since the amplitudes or powers are not reliable in MESA (Kane \& Trivedi, 1982), the periodicities $\mathrm{T}_{\mathrm{k}}(\mathrm{k}=1, \mathrm{n}$; where $\mathrm{n}$ is the number of periodicities) were used in an MRA programme to obtain their corresponding amplitudes. The MRA programme is inside the MESA programme.

\section{Results and discussion}

Fig. 2 shows the plot of the normalised rainfall values for the northern and southern zones of Ghana as well as for the entire country. The figure generally showed floods in 1962, 1963 and 1968; and droughts in 1977, 1983, 1992 and 1998. Similar plots, not presented here for the various stations revealed that these were widespread.

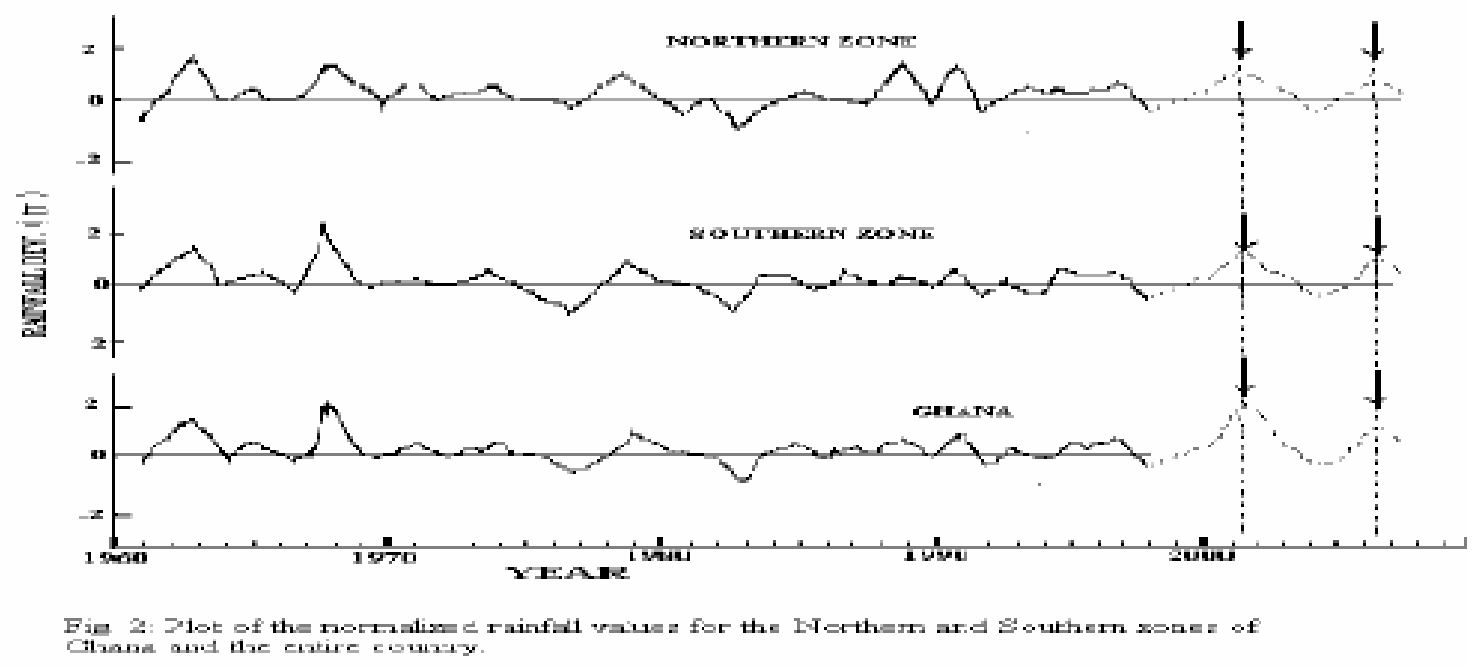

The spectra for the two zones were identical to that of the entire country (Fig. 3). The spectra for Ghana is, therefore, quite representative of either zone. The perio-dicities detected in the Ghana spectra by the MESA programme were 2.2, 2.4, 2.7, 3.3, 4.2, 5.6 and 8.5 years. Similar periodicities have been detected in rainfall series throughout equatorial and Southern Africa (Nicholson \& Entekhabi, 1986). The range of periodicities of 2.2-2.4 years indicated the presence of a quasi-biennial oscillation in the rainfall time series in Ghana. 


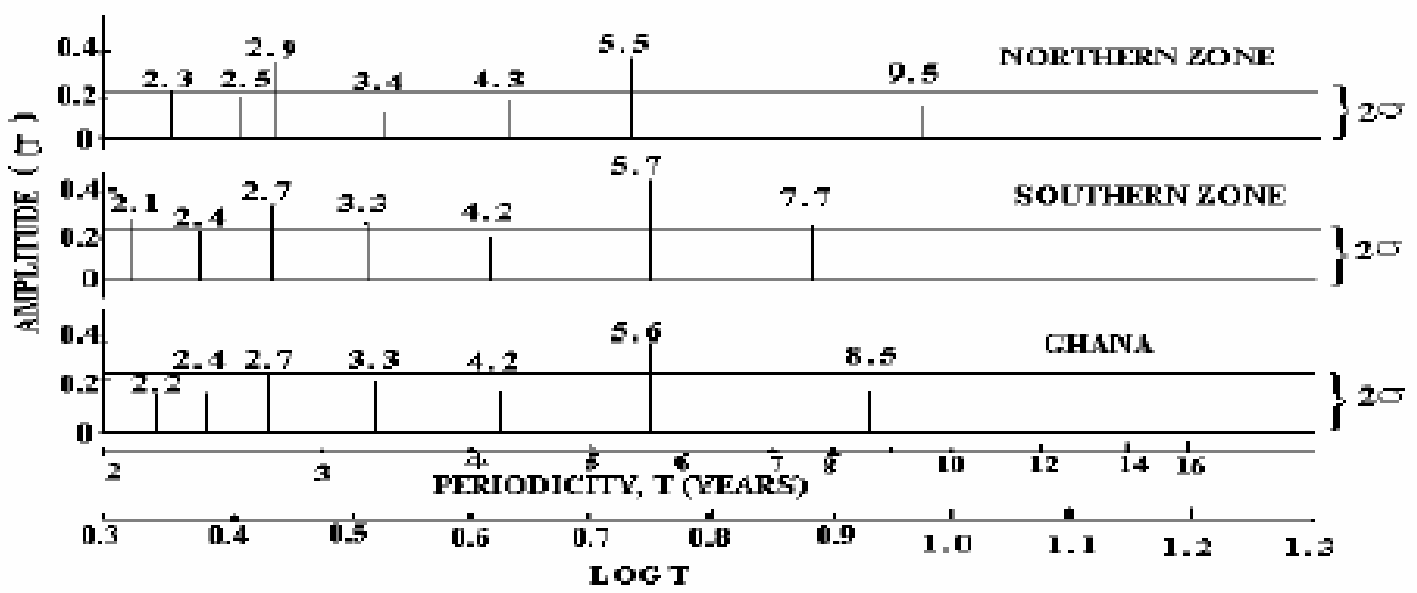

Fig. 3: Spectra showing amplitudes and their corresponding periodicities for the lorthem and Southem zones of Ghana and the entire country.

The amplitude corresponding to the periodicity of 5.6 years was far greater than $2 \sigma$, (the standard error, s being 0.11). The periodicity was, therefore, highly significant. The 2.7-year periodicity was of a borderline significance, while the rest, all of whose amplitudes were less than $2 \sigma$, were insignificant (Fig. 3 ). The extreme positive rainfall deviations resulting in major floods could be attributed to the periods when the two major oscillations, of periodicities 5.6 and 2.7 years, interfere constructively. Similarly, the extreme negative rainfall deviations resulting in major droughts could be attributed to the periods when the two major oscillations, interfere destructively.

The most recent floods or excess rains (positive deviation) within the 1961-1998 period occurred in 1996. Based on the highly significant periodicity of 5.6 years, the next possible floods after the 1996 floods were predicted to occur during the year 2001 or 2002. The current widespread floods in Ghana have proved this prediction right. On the same premise as stated above, the next major floods in Ghana may be predicted to occur in year 2006 or 2007. Similarly, with the last major drought occurring in 1998, the next major drought might occur in year 2003 or 2004.

\section{Conclusion}

- Rainfall extremes in Ghana showed a highly significant periodicity of 5.6 years.

- The next major floods predicted, by the MESA programme, to occur in Ghana after the 1996 floods were to be in 2001 or 2002. The current widespread floods in Ghana have proved this prediction right.

- MESA is a powerful tool for the prediction of floods in Ghana.

- After the floods of 2001 or 2002, the MESA programme predicts the next major floods in Ghana to occur in the year 2006 or 2007.

Every effort should be made to minimize such risks as deaths, loss of property and damage to infrastructure, associated with floods in Ghana. The use of MESA to accurately predict these floods would not only help in minimizing these risks, but would also save the nation the scare resources needed for development.

\section{Acknowledgement}

The authors are indebted to Dr R. P. Kane of Instituto de Pesquisas Espacias (INPE), Brazil, for providing them with the Maximum Entropy Spectral Analysis (MESA) programme used for analysing the data. They are also 


\section{West Africa Journal of Applied Ecology (WAJAE) -ISSN: 0855-4307 \\ Volume 9 (Jan - Jun 2006) \\ www.wajae.org}

grateful to the Ghana Meteorological Services Department, Accra, for providing the rainfall data used in the study.

\section{References}

Bevington P. R. (1969). Data reduction and Error Analysis for the Physical Sciences, pp.164-174. McGraw-Hill, New York.

Blackman R. B. and Tukey J. M. (1958). The Measurements of Spectra, Dover, New York.

Bunting A. H., Dennett M. D., Elston J. and Milford J. R. (1976). Rainfall trends in the West African Sahel. Q. J. R. Meteorol. Soc. 102: 59-64.

Burg J. P. (1967). Maximum entropy spectral analysis. Paper presented at the 37th Meeting, Society of Exploration Geophysics. Oklahoma City, October.

Faure H. and Gac J. Y. (1981). Will the Sahelian drought end in 1985? Nature 291: 475-478.

Hulme M. (1994). Century-scale time series of regional rainfall anomalies in Africa. In Trends'93, A Compendium of Data on Global change,ornl/cdiac-65. (T. A. Boden, D. P. Kaiser, Sepanski, R. J. and F. W. Stoss, ed.), pp. 964-973. Carbon Dioxide Information Analysis Centre, Oak Ridge National Laboratory, Oak Ridge, Tenn., USA.

Kane R. P. (1979). Maximum entropy spectral analysis of some artificial samples. J. Geophys. 84 (A3): 965-966.

Kane R. P. and Trivedi N. B. (1982). Comparison of maximum entropy spectral analysis (MESA) and least-square linear prediction (LSLP) methods for some artificial samples. Geophysics 47: 1731-1736.

Kane R. P. and Trivedi N. B. (1986). Are droughts predictable? Climatic Change 8: 209-223.

Lansford H. (1977). Tree-rings; predictors of drought? Weatherwise 32: 194-199.

Nicholls N. (1980). Long-range weather forecasting: value, status and prospects, Rev. Geophys. Space Phys. 18: 771-788.

Nicholson S. E. (1980). The nature of rainfall fluctuations in sub-tropical West Africa Mon. Wea. Rev. 108(4): 473-475.

Nicholson S. E. (1994). Century-scale series of standardised annual departures of African rainfall. In Trends '93, A Compendium of Data on Global change, ORNL/CDIAC-65. (T. A. Boden, D. P. Kaiser, R. J. Sepanski and F. W. Stoss, ed.), pp. $952-962$. Carbon Dioxide Information Analysis Centre, Oak Ridge national Laboratory, Oak Ridge, Tenn., USA.

Nicholson S. E. and Entekhabi D. (1986). The quasi-periodic behaviour of rainfall variability in Africa and its relationship to the Southern Oscillation. Arch. Meteor. Geophys. Bioclim. Ser. A34: 311-348.

Ogallo L. (1979). Rainfall variability in Africa. Mon. Wea. Rev. 107: 1133-1139.

Quinn W. H., Neal V. T. and Antunes de Mayolo S. E. (1987). El Niño occurrences over the past four and a half centuries. J. Geophys. Res. 92: 14449-14461.

Rodhe H. and Virji H. (1976). Trends and periodicities in East African rainfall data. Mon. Wea. Rev. 104: 307-315.

Tabony R. C. (1979). Spectral and filter analysis of long-period rainfall records in England and Wales. Meteor. Magazine, 108: 97-118.

Tyson P. D., Dyer R. G. J. and Mametse M. N. (1975) Secular changes in South African rainfall: 1880 to 1972. Q. J.R. Met. Soc. 101: 817-832.

Ulrych T. J. and Bishop T. N. (1975). Maximum entropy spectral analysis and autoregressive decomposition, Rev. Geophys. 13: 183200.

Winstanley D. (1973a). Recent rainfall trends in Africa, the Middle East and India. Nature. 243: 464-465.

Winstanley D. (1973b). Rainfall Patterns and General Atmospheric circulation. Nature. 245: 190-194.

Wright P. B. (1977). The southern oscillation patterns and mechanisms of the teleconnections and the persistence. Rep. HIG 77-13, Hawaii Institute of Geophysics, Univ. of Hawaii. 107 pp. 